\title{
Canadian medical residents report pervasive harassment, crushing workloads
}

\author{
n Cite as: CMAJ 2018 November 19;190:E1371. doi: 10.1503/cmaj.109-5678
}

Posted on cmajnews.com on Oct. 31, 2018.

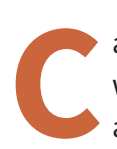
anadian medical residents are working under immense pressure and face pervasive abuse on the job, according to a national survey by Resident Doctors of Canada. Most trainees said they would give up some professional freedom for better benefits, and nearly one in five regret their choice of career.

"Our survey uncovered myriad challenges facing resident doctors, including intimidation and harassment, presenteeism, burnout and depression," according to survey team co-chair Dr. Melanie Bechard. Of the 10,091 residents invited to participate by email, 833 completed the survey for a response rate of $8.3 \%$.

More than three-quarters of the residents surveyed said they had experienced some form of harassment and intimidation in the preceding year. Almost all were on the receiving end of inappropriate comments. About one in five reported being given work as punishment or having privileges and opportunities taken away, and more than 1 in 10 said they were sexually harassed or touched inappropriately.

Patients were the most common source of abuse, with $77.1 \%$ of trainees reporting an incident in the previous year. However, they also reported harassment and intimidation from allied health professionals (55.3\%), staff physicians (51.9\%), other residents (35.7\%) and program directors (7.6\%). The highest rate of bullying was reported by surgical residents (85.7\%); the lowest in family medicine (69.5\%). Residents believed gender was a factor in more than one-third of incidents.

Nearly 9 of 10 trainees did not use their institutions' resources to address harassment and intimidation, and among the few who did, three in five felt the resources were inadequate. Harassment was also cited among the top reasons residents considered transferring to another training program.

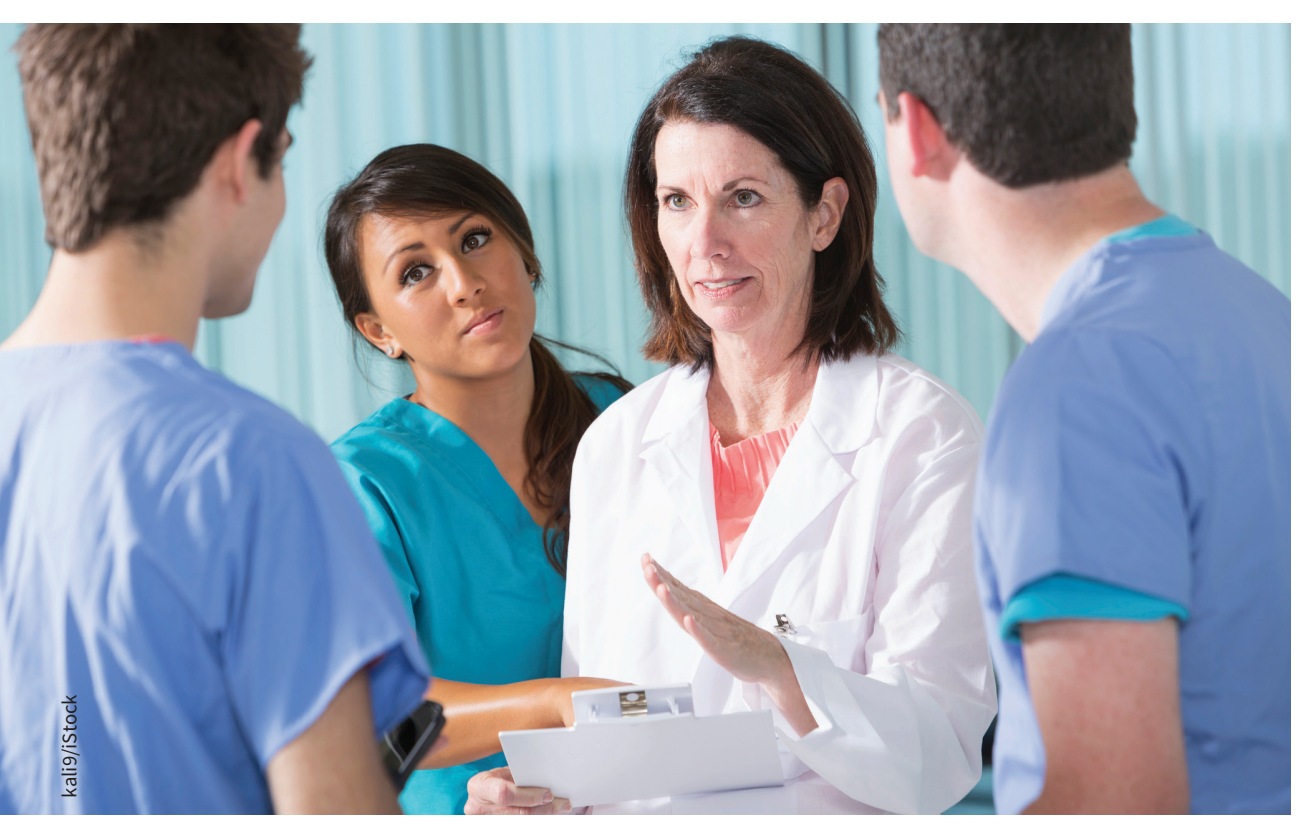

Three of four residents surveyed said they've experienced harassment or intimidation on the job.
Most residents reported high levels of resilience but $51.9 \%$ still reported symptoms of burnout, such as physical and emotional exhaustion. More than 1 in 10 reported feeling down, depressed or hopeless most days in the preceding two weeks. More than one in seven had thought about suicide in the last year; $2.5 \%$ had seriously considered it.

The culture of medicine and residents' lack of control over their schedules were cited as the biggest barriers to seeking mental health care. Nearly two-thirds of residents said their work schedules didn't leave them enough time for a personal life or family. Most work 60 or more hours a week, and close to one in five work more than 80 hours a week. Ninety-one percent of residents said they came to work sick at least sometimes, and about $20 \%$ admitted to making medical errors because of fatigue.

Looking back on their career choice, nearly one in five said it was unlikely or very unlikely that they would choose medicine again. According to Bechard, the new generation of resident doctors "envision a different practice environment from decades past." Residents indicated a desire for national licensure and a preference for salaried payment models, "which may serve as an indicator of what is to come."

More than half of residents said they would trade some clinical autonomy for a salaried position that included health benefits, pension, vacation time and other benefits. Although $65 \%$ of residents intend to stay and practise in the province where they are training, more than half of those not planning to locum elsewhere in the country would do so if no additional licence applications were required.

Lauren Vogel, CMAJ 